\title{
Instrumentos para a avaliação do nível de consciência em adultos e idosos: revisão integrativa
}

\author{
Instruments for assessing level of consciousness in adults and the elderly: integrative review \\ Instrumentos para evaluar el nivel de conciencia en adultos y ancianos: revisión integradora
}

\author{
Fabiana Cristina Pires ${ }^{\oplus}$, Luana Vilela e Vilaça ${ }^{\oplus}$, Caroline Bueno de Moraes Pereira ${ }^{\circledR}$, \\ Mariana Torreglosa Ruiz' $\odot$, Rosali Isabel Barduchi Ohl" ${ }^{\circledR}$, Suzel Regina Ribeiro Chavaglia' ${ }^{\circ}$
}

'Universidade Federal do Triangulo Mineiro, Uberaba, MG, Brasil; "Universidade Federal de São Paulo, São Paulo, SP, Brasil

\begin{abstract}
RESUMO
Objetivo: identificar na literatura evidências científicas sobre os instrumentos que avaliam o nível de consciência de pacientes adultos e idosos. Método: revisão integrativa de literatura, realizada em fevereiro de 2020, com artigos publicados entre 2010 e 2020, nas fontes de informação: LILACS, CINAHL, PubMed\%/Medline, Web of Science, EBSCO e Scopus. Adotou-se o aplicativo Rayyan para a seleção dos estudos. Resultados: identificaram-se 884 artigos, destes, 19 atenderam aos critérios de inclusão. Foram identificadas oito escalas de avaliação. A maioria delas avalia os itens: abertura ocular, resposta motora, resposta verbal, padrão respiratório e reflexos tronco encefálicos. A escala Coma Recovery Scale-Revised foi a mais utilizada e a escala Full Outline of Unresponsiveness, considerada a mais completa para avaliar a consciência. Conclusão: verificou-se que as escalas possuem semelhança em seus domínios e mecanismos de avaliação e são confiáveis e efetivas para mensurar o nível de consciência de adultos e idosos.
\end{abstract}

Descritores: Estado de consciência; Pacientes; Adulto; Idoso; Questionários.

\begin{abstract}
Objective: to identify, in the literature, scientific evidence on the instruments that assess level of consciousness in adult and elderly patients. Method: this integrative literature review was carried out in February 2020, with articles published between 2010 and 2020, in the information sources: LILACS, CINAHL, PubMed ${ }^{\otimes} /$ Medline, Web of Science, EBSCO and Scopus. Studies were selected using the application Rayyan. Results: 884 articles were identified, of which 19 met the inclusion criteria. Eight rating scales were identified, most evaluating the items: eye opening, motor response, verbal response, breathing pattern and brainstem reflexes. The Coma Recovery Scale-Revised was the most used and the Full Outline of Unresponsiveness scale was considered the most complete to assess awareness. Conclusion: the scales were found to share similar domains and evaluation mechanisms and to be reliable and effective for measuring level of consciousness in adults and the elderly.

Descriptors: Consciousness; Patients; Adult; Aged; Questionnaires.

\section{RESUMEN}

Objetivo: identificar en la literatura evidencia científica sobre los instrumentos que evalúan el nivel de conciencia de pacientes adultos y ancianos. Método: revisión integradora de la literatura, realizada en febrero de 2020, con artículos publicados entre 2010 y 2020, en las fuentes de información: PubMed /Medline, LILACS, CINAHL, Web of Science, EBSCO y Scopus. Se adoptó la aplicación Rayyan para seleccionar los estudios. Resultados: Se identificaron 884 artículos, de los cuales 19 cumplieron con los criterios de inclusión. Se identificaron ocho escalas de calificación. La mayoría de ellos evalúa los ítems: apertura ocular, respuesta motora, respuesta verbal, patrón de respiración y reflejos del tronco encefálico. La escala Coma Recovery ScaleRevised fue la más utilizada y la escala Full Outline of Unresponsiveness fue considerada la más completa para evaluar la conciencia. Conclusión: se verificó que las escalas tienen similitudes en sus dominios y mecanismos de evaluación y son confiables y efectivas para medir el nivel de conciencia de adultos y ancianos.
\end{abstract}

Descriptores: Estado de conciencia; Pacientes; Adulto; Anciano, Cuestionarios.

\section{INTRODUÇÃO}

A identificação do nível de consciência constitui-se um dos parâmetros mais importantes da avaliação neurológica e é conceituada como a análise do grau de alerta comportamental que o indivíduo apresenta, refletido por estruturas primordiais do Sistema Nervoso Central (SNC), como o tronco cerebral e outras áreas encefálicas ${ }^{1,2}$. A alteração do nível de consciência é considerada um dos primeiros sinais de modificação do estado clínico que um paciente adulto ou idoso apresenta diante de uma enfermidade ${ }^{3}$.

Desta forma, avaliar o nível de consciência é um cuidado indispensável para a assistência de adultos e idosos, por permitir à equipe de saúde determinar a gravidade ou mudança clínica desta população, por meio de suas alterações comportamentais, compreendidas entre estar completamente alerta até o coma profundo ${ }^{4}$.

Autora correspondente: Suzel Regina Ribeiro Chavaglia. E-mail: suzel.ribeiro@yahoo.com.br

Editora Científica: Cristiane Helena Gallasch; Editora Associada: Cintia Silva Fassarella 
Para a enfermagem, a avaliação do nível de consciência é considerada um dos pontos de partida fundamentais para a sistematização da assistência, de maneira objetiva, reprodutível e universal, viabilizada pela adoção de instrumentos de avaliação clínica ${ }^{5}$. Desta forma, esta prática assistencial proporciona monitorar o paciente, executar intervenções, definir diagnósticos e, por isso, não deve ser realizada, baseando-se em julgamentos imprecisos, condicionados a uma avaliação subjetiva ${ }^{4,5}$.

Neste contexto, a adoção de escalas de avaliação do nível de consciência, de fácil aplicação e capazes de facilitar a identificação rápida de disfunções ou mudanças nos parâmetros neurológicos representam importante progresso para garantir uma terapêutica adequada para cada caso $^{4-6}$.

Nas últimas décadas, inúmeras escalas foram elaboradas e testadas para avaliar clinicamente o nível de consciência de pacientes adultos e idosos, com destaque para a Glasgow Coma Scale ${ }^{6}$, um instrumento amplo que conduz a identificação do estado de consciência de um paciente, gera um escore e subsidia a tomada de decisão clínica mais apropriada. No entanto, cabe ressaltar que as escalas de avaliação do nível de consciência, em geral, possuem o intuito de padronizar o acompanhamento da evolução clínica, a comunicação efetiva entre as equipes de saúde e identificar, de maneira efetiva, este parâmetro neurológico, com segurança e confiabilidade por meio de referenciais teóricos fidedignos e submissão a rigorosos processos de validação ${ }^{6,7}$.

As escalas e outros instrumentos para realização do exame neurológico na avaliação do nível de consciência de pacientes graves significam um marcante avanço para a garantia da qualidade da assistência prestada. Para tanto, tornase necessário que essas escalas apresentem fácil aplicabilidade e que permitam o reconhecimento precoce de alterações e/ou variações dos parâmetros neurológicos do paciente ${ }^{4,5}$.

Apesar de já ter sido desenvolvida uma variabilidade de constructos que se predispõem a avaliar o nível de consciência de um indivíduo, há uma importante lacuna científica quanto à exploração de estudos que apresentam com clareza os principais instrumentos existentes, para este fim $^{8}$.

Soma-se a este cenário, a incipiência de manuscritos que abordam os domínios e a estrutura de cada escala, os mecanismos de avaliação do nível de consciência e a sua efetividade, mesmo diante da necessidade de compreenderse o estado neurológico de um paciente adulto, para uma tomada de decisão assertiva em sua assistência e tratamento $^{7,8}$, e ainda, ao número limitado de escalas que cumpram este fim, validadas para o português do Brasil.

Esta escassez de produção cientifica sobre a temática, pode dificultar a determinação do instrumento mais confiável e adequado para este propósito, o que implica negativamente a qualidade do atendimento prestado ao paciente e em relação à sua segurança ${ }^{8}$.

Essas escalas, antes de serem consideradas adequadas para sua aplicação, necessitam fornecer informações específicas, pertinentes e elucidativas para a avaliação em saúde, a fim de que as mensurações possam fornecer resultados cientificamente significativos por meio da análise de confiabilidade e validade desses instrumentos ${ }^{8}$.

Diante da relevância da avaliação do nível de consciência de pacientes adultos e idosos, baseada em instrumentos validados e abrangentes e da necessidade de aprofundamento científico neste âmbito, surge o seguinte questionamento: "Quais são os instrumentos que avaliam o nível de consciência de pacientes adultos e idosos em ambiente intra-hospitalar?". Este estudo visa a identificar na literatura evidências científicas sobre os instrumentos que avaliem o nível de consciência de pacientes adultos e idosos.

\section{MÉTODO}

Trata-se de uma revisão integrativa da literatura, tipo de estudo que possibilita a síntese e análise do conhecimento científico já produzido sobre determinada temática, sustentada pelo Statement for Reporting Systematic Reviews and Meta-Analyses of Studies (PRISMA), referencial teórico-metodológico composto de um checklist de 27 itens e um fluxograma de quatro etapas, que suportam a qualidade de estudos de revisão. Para a elaboração da revisão percorreram-se 6 etapas: (1) definição da questão de pesquisa; (2) estabelecimento dos critérios de inclusão e exclusão com a busca na literatura; (3) definição das informações a serem extraídas dos estudos; (4) avaliação dos estudos incluídos; (5) interpretação dos resultados e (6) síntese dos dados ${ }^{9}$.

Na primeira etapa, identificou-se o tema que abordou os instrumentos de avaliação do nível de consciência de pacientes adultos e idosos e seus mecanismos de avaliação, formulando-se a pergunta de pesquisa embasada na estratégia Population - Interest Phenomenon - Context (PICo $)^{10}$ : "Quais são os instrumentos que avaliam o nível de consciência de pacientes adultos e idosos em ambiente intra-hospitalar?". O acrônimo "P" - população, foi representado por pacientes adultos e idosos; o acrônimo "I" - intervenção, configurou-se pela identificação dos instrumentos de avaliação do nível de consciência; e o acrônimo "Co" - contexto, foi representado por ambiente intra-hospitalar. 
Na segunda etapa definiram-se os critérios de inclusão: estudos primários que respondessem à pergunta de pesquisa, publicados entre os anos de 2010 e 2020 e sem delimitação de idioma. Excluíram-se estudos do tipo revisão, teses, dissertações, artigos de opinião, comentários, ensaios, notas prévias, manuais, livros, capítulos de livros, obituários e, artigos que abordassem instrumentos para avaliar o nível de consciência de outras populações que não fossem configuradas por adultos e idosos e em ambiente intra-hospitalar.

Utilizaram-se as seguintes fontes de informações: US National Library of Medicine National Institutes Database Search of Health (PubMed ${ }^{\circledR} /$ Medline), Literatura Latino-americana e do Caribe em Ciências da Saúde (LILACS), Cumulative Index to Nursing and Allied Health Literature (CINAHL), Web of Science, EBSCO Information Sevices e SciVerse Scopus.

A busca dos estudos ocorreu no mês de fevereiro de 2020 por meio dos descritores em saúde disponíveis no Portal de Descritores em Ciências da Saúde (DeCS) na Biblioteca Virtual da Saúde (BVS) e pelos descritores controlados do Medical Subject Headings, identificados junto à respectiva estratégia de busca, específicos para cada base de dados selecionada, validados por uma bibliotecária, e descritos, a seguir.

Na PubMed /MEDLINE e Scopus, foram identificados os descritores controlados no Medical Subjects Headings (MeSH), na língua inglesa: Consciousness Disorders; Patients; Adult e adotada a palavra-chave: Scales. Utilizou-se a estratégia: (“Consciousness Disorders" AND [scales] AND patients AND adult).

$\mathrm{Na}$ LILACS, os descritores controlados estavam presentes nos Descritores de Ciências da Saúde (DeCS), em português: Transtornos da Consciência; Pacientes; Adulto. Adotou-se a palavra-chave: Escala e suas versões em ingles e espanhol. Realizou-se a estratégia: ("Transtornos da Consciência" OR "Consciousness Disorders" OR "Trastornos de la Conciencia" AND [escalas OR scales] AND pacientes OR patients AND adulto OR adult).

$\mathrm{Na}$ CINAHL identificou-se os descritores controlados em Títulos/Assuntos, na língua inglesa: Consciousness Disorders; Patients; Adult. Adotou-se a palavra-chave: Escala. Empregou-se a estratégia: ("Consciousness Disorders" AND [scales] AND patients AND adult).

Na web of science utilizaram-se os descritores controlados em inglês: Consciousness Disorders; Patients; Adult e a palavra-chave: Escala. Adotou-se a estratégia: TS=(“Consciousness Disorders” AND [scales] AND patients AND adult).

$\mathrm{Na}$ EBSCO os descritores controlados estavam presentes no Descritores de Ciências da Saúde (DeCS), em português: Transtornos da Consciência; Pacientes; Adulto. Adotou-se a palavra-chave: Escala e suas versões em ingles e espanhol. Realizou-se a estratégia: ("Transtornos da Consciência" OR “Consciousness Disorders" OR "Trastornos de la Conciencia" AND [escalas OR scales] AND pacientes OR patients AND adulto OR adult).

Na Scopus utilizaram-se os descritores controlados em inglês: Consciousness Disorders; Patients; Adult e adotada a palavra-chave: Scales. Procedeu-se a estratégia: ("Consciousness Disorders" AND [scales] AND patients AND adult).

Para selecionar os estudos por meio dos critérios de inclusão e exclusão, procedeu-se primeiramente, à leitura de títulos e resumos, viabilizada pelo programa de revisão gratuito da web de versão única, chamado Rayyan Qatar Computing Research Institute (Rayyan QCRI), encontrado no link: https://rayyan.qcri.org, que elimina artigos duplicados, agiliza a triagem inicial, usando um processo fidedigno de semiautomação, e incorpora alto nível de usabilidade e eficácia ao processo ${ }^{11}$. Após a seleção por títulos e resumos, dez estudos que ocasionaram divergência entre os pesquisadores foram entregues a um terceiro, responsável por tomar a decisão de inclusão ou exclusão e, em seguida, realizou-se a leitura na íntegra para definição da amostra final.

$\mathrm{Na}$ terceira etapa, definiram-se as informações a serem extraídas de cada estudo, utilizando-se os critérios de um instrumento validado de Ursi e Galvão ${ }^{12}$, cuja abordagem inclui: estudo, periódico, objetivo, tipo de estudo, resultados/conclusões e classificação do nível de evidência.

Para classificar o nível de evidência dos estudos selecionados, foram utilizadas as categorias da Agency for Healthcare Research and Quality (AHRQ), que abrangem seis níveis: nível 1: meta-análise de múltiplos ensaios clínicos controlados e randomizados; nível 2: estudos individuais com delineamento experimental; nível 3: estudos quase experimentais; nível 4: estudos descritivos (não experimentais) ou abordagem qualitativa; nível 5: relatos de caso ou experiência; nível 6: opiniões de especialistas ${ }^{13}$. Na quarta etapa ocorreu a leitura individual na íntegra dos estudos incluídos, a avaliação crítica e interpretação dos resultados com a síntese de conhecimento.

\section{RESULTADOS}

A presente revisão integrativa identificou a priori, 884 estudos e demonstrou-se sua seleção, por meio do Preferred Reporting Items for Systematic Reviews and Meta-Analyses (PRISMA)14, conforme apresentado na figura 1, a seguir. 


\section{IDENTIFICAÇÃO DE NOVOS ESTUDOS POR MEIO DE BANCOS DE DADOS E REGISTROS}

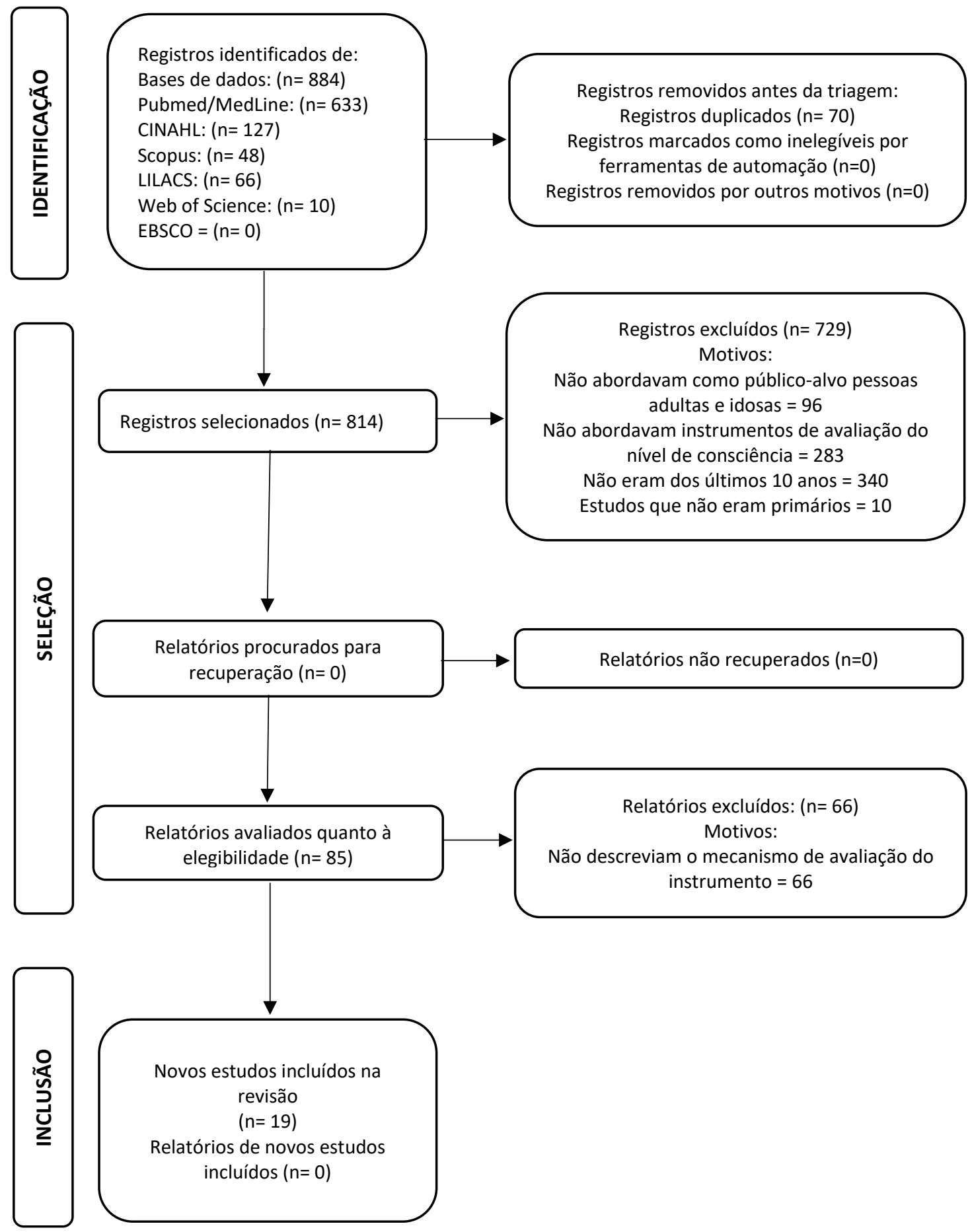

FIGURA 1: Apresentação da seleção dos estudos que compuseram a amostra da presente revisão integrativa, segundo Preferred Reporting Items for Systematic Reviews and Meta-Analyses (PRISMA). Uberaba, MG, Brasil, 2020.

Fonte: Page et al., $2020^{14}$.

CINAHL: Cumulative Index to Nursing and Allied Health Literature; LILACS: Literatura Latino-Americana e do Caribe em Ciências da Saúde.

A caracterização dos estudos, incluídos na amostra, é apresentada na Figura 2, apresentada a seguir. 


\begin{tabular}{|c|c|c|c|}
\hline ESTUDO/ PERIÓDICO & OBJETIVOS & $\begin{array}{l}\text { TIPO DE } \\
\text { ESTUDO/ NÍVEL } \\
\text { DE EVIDÊNCIA* }\end{array}$ & RESULTADOS/CONCLUSÃO \\
\hline $\begin{array}{l}\text { E15/IEEE Trans. Neural } \\
\text { Syst. Rehabil. Eng. }{ }^{15}\end{array}$ & $\begin{array}{l}\text { Propor uma nova interface de } \\
\text { computador, baseada em estímulos } \\
\text { audiovisuais para avaliar o nível de } \\
\text { consciência. }\end{array}$ & $\begin{array}{l}\text { Estudo } \\
\text { Experimental } \\
\text { Randomizado/2 }\end{array}$ & $\begin{array}{l}\text { A avaliação baseada na nova interface de } \\
\text { computador é um objeto mais sensível quando } \\
\text { comparado com a escala de avaliação clínica em } \\
\text { pacientes. }\end{array}$ \\
\hline $\begin{array}{l}\text { E16/J. Pain Symptom } \\
\text { Manage. }{ }^{16}\end{array}$ & $\begin{array}{l}\text { Investigar o Índice de Complexidade } \\
\text { (ICs). }\end{array}$ & $\begin{array}{l}\text { Estudo } \\
\text { Observacional } \\
\text { Retrospectivo/4 }\end{array}$ & $\begin{array}{l}\text { Identificou-se uma correlação entre ICs e a Coma } \\
\text { Recovery Scale-Revised (CRS-R). }\end{array}$ \\
\hline E17/Neurol. Sci. ${ }^{17}$ & $\begin{array}{l}\text { Avaliar o nível de consciência de } \\
\text { pacientes com a Nociception Coma } \\
\text { Scale-Revised (NCS-R) e a CRS-R. }\end{array}$ & $\begin{array}{l}\text { Estudo de } \\
\text { Correlação/ } 4\end{array}$ & $\begin{array}{l}\text { A correlação entre os escores NCS-R e CRS-R pode } \\
\text { ser útil na avaliação clínica de pacientes. }\end{array}$ \\
\hline E18/ Neurol. Sci. ${ }^{18}$ & $\begin{array}{l}\text { Avaliar a validade diagnóstica da Brief } \\
\text { Post-Coma Scale (BP-CS) comparando- } \\
\text { a com a Coma Recovery Scale Revised. }\end{array}$ & $\begin{array}{l}\text { Estudo } \\
\text { Experimental/3 }\end{array}$ & $\begin{array}{l}\text { A BP-CS demonstrou correlações significativas com } \\
\text { as escalas comumente usadas em pessoas com } \\
\text { distúrbio da consciência. }\end{array}$ \\
\hline $\begin{array}{l}\text { E19/Neuropsychol. } \\
\text { Rehabil. }{ }^{19}\end{array}$ & Validar a versão italiana da NCS-R. & $\begin{array}{l}\text { Estudo } \\
\text { Metodológico/6 }\end{array}$ & A NCS-R manteve as propriedades psicométricas. \\
\hline E20/Neurocrit. Care 20 & $\begin{array}{l}\text { Validar a versão persa da escala Full } \\
\text { Outline of Unresponsiveness (FOUR). }\end{array}$ & $\begin{array}{l}\text { Estudo } \\
\text { Metodológico/6 }\end{array}$ & $\begin{array}{l}\text { A FOUR é uma escala confiável e válida para avaliar } \\
\text { pacientes inconscientes. }\end{array}$ \\
\hline E21/Plos One 21 & $\begin{array}{l}\text { Determinar os sinais clínicos que levam } \\
\text { a uma avaliação precisa da } \\
\text { consciência. }\end{array}$ & \begin{tabular}{|l} 
Estudo \\
Descritivo/6
\end{tabular} & $\begin{array}{l}\text { Houve associação da pontuação Motor Behaviour } \\
\text { Tool (MBT) e CRS-R com o nível de consciência e a } \\
\text { predição do desfecho. }\end{array}$ \\
\hline E22/Clin. J. Pain. ${ }^{22}$ & Avaliar a utilidade clínica da NCS-R. & \begin{tabular}{|l} 
Estudo \\
Descritivo/6
\end{tabular} & $\begin{array}{l}\text { A NCS-R é uma ferramenta útil para avaliar o nível } \\
\text { de consciência. }\end{array}$ \\
\hline $\begin{array}{l}\text { E23/BMC Neurology. } \\
23\end{array}$ & Analisar a efetividade da CRS-R. & $\begin{array}{l}\text { Estudo } \\
\text { Observacional/4 }\end{array}$ & $\begin{array}{l}\text { O uso da CRS-R pode minimizar o risco de } \\
\text { classificação incorreta do nível de consciência. }\end{array}$ \\
\hline E24/BMC Neurology 24 & $\begin{array}{l}\text { Validar a escala FOUR na versão } \\
\text { chinesa. }\end{array}$ & $\begin{array}{l}\text { Estudo } \\
\text { Metodológico/6 }\end{array}$ & $\begin{array}{l}\text { A escala FOUR é confiável para avaliar o nível de } \\
\text { consciência na lesão cerebral. }\end{array}$ \\
\hline $\begin{array}{l}\text { E25/Arch.PhysMed. } \\
\text { Rehabil. }{ }^{25}\end{array}$ & $\begin{array}{l}\text { Avaliar a validade interna e a } \\
\text { confiabilidade da CRS-R. }\end{array}$ & $\begin{array}{l}\text { Estudo } \\
\text { Observacional/4 }\end{array}$ & $\begin{array}{l}\text { A CRS-R é uma ferramenta de medição } \\
\text { psicometricamente sólida e robusta. }\end{array}$ \\
\hline E26/J. Neurol. ${ }^{26}$ & $\begin{array}{l}\text { Utilizar o eletroencefalografia para } \\
\text { avaliar o nível de consciência. }\end{array}$ & $\begin{array}{l}\text { Estudo } \\
\text { Descritivo/6 }\end{array}$ & $\begin{array}{l}\text { Os parâmetros do eletroencefalografia } \\
\text { e a do CRS-R fornecem significante relevância } \\
\text { clinica. }\end{array}$ \\
\hline $\begin{array}{l}\text { E27/Arch. Phys. Med. } \\
\text { Rehabil. }{ }^{27}\end{array}$ & $\begin{array}{l}\text { Descrever as características clínicas e o } \\
\text { padrão evolutivo de pacientes com } \\
\text { lesão cerebral. }\end{array}$ & $\begin{array}{l}\text { Estudo de } \\
\text { Coorte/4 }\end{array}$ & $\begin{array}{l}\text { A CRS-R estabelece um prognóstico imediato para } \\
\text { a estado minimamente consciente e o estado } \\
\text { vegetativo. }\end{array}$ \\
\hline $\begin{array}{l}\text { E28/Anaesth. } \\
\text { Intensive Care } 28\end{array}$ & Investigar a utilidade da escala FOUR. & $\begin{array}{l}\text { Estudo } \\
\text { Observacional/4 }\end{array}$ & $\begin{array}{l}\text { A FOUR é útil para avaliar pacientes com Acidente } \\
\text { Vascular Cerebral agudo. }\end{array}$ \\
\hline $\begin{array}{l}\text { E29/Neurologic Critical } \\
\text { Care }^{29}\end{array}$ & $\begin{array}{l}\text { Examinar a confibialidade } \\
\text { interobservador da escala FOUR. }\end{array}$ & $\begin{array}{l}\text { Estudo } \\
\text { Metodológico/6 }\end{array}$ & $\begin{array}{l}\text { A FOUR mostrou-se excelente e confiavel nos cinco } \\
\text { diferentes hospitais. }\end{array}$ \\
\hline $\begin{array}{l}\text { E30/Intern. Emerg. } \\
\text { Med. }{ }^{30}\end{array}$ & $\begin{array}{l}\text { Validar a versão italiana da escala } \\
\text { FOUR. }\end{array}$ & $\begin{array}{l}\text { Estudo } \\
\text { Metodológico/6 }\end{array}$ & $\begin{array}{l}\text { A FOUR fornece mais detalhes neurológicos do que } \\
\text { o Glasgow Coma Scale (GCS). }\end{array}$ \\
\hline E31/Neurocrit. Care ${ }^{31}$ & Comparar a escala FOUR e GCS. & $\begin{array}{l}\text { Estudo } \\
\text { Experimental/3 }\end{array}$ & $\begin{array}{l}\text { A FOUR é válida quando comparada com a GCS na } \\
\text { predição de prognóstico. }\end{array}$ \\
\hline E32/Brain Injury ${ }^{32}$ & Validar a versão italiana da CRS-R. & $\begin{array}{l}\text { Estudo } \\
\text { Metodológico/6 }\end{array}$ & $\begin{array}{l}\text { A CRS-R discrimina pacientes em estado vegetativo } \\
\text { e minimamente consciente. }\end{array}$ \\
\hline E33/Pain 33 & Validar a NCS-R. & \begin{tabular}{|l|} 
Estudo \\
Metodológico/6
\end{tabular} & $\begin{array}{l}\text { A NCS-R constitui uma ferramenta sensível para } \\
\text { avaliar a nocicepção. }\end{array}$ \\
\hline
\end{tabular}

FIGURA 2: Caracterização dos estudos incluídos na revisão integrativa. Uberaba, MG, Brasil, 2020.

Fonte: Elaborado pelo autor, 2020.

*Agency for Healthcare Research and Quality, 2016 
A Figura 3, a seguir, apresenta as principais escalas, o mecanismo utilizado pelos instrumentos para avaliar a consciência de adultos e idosos e os estudos que abordam as escalas.

\begin{tabular}{|c|c|c|}
\hline Instrumento & Método & Estudos \\
\hline $\begin{array}{l}\text { Coma Recovery Scale- } \\
\text { Revised (CRS-R) }\end{array}$ & $\begin{array}{l}\text { A CRS-R é composta de } 23 \text { itens, agrupados em seis subescalas: auditivo, visual, motor, } \\
\text { oromotor, comunicação e excitação. A pontuação mais baixa em cada subescala representa } \\
\text { atividade reflexiva; o mais alto representa comportamentos mediados pela entrada } \\
\text { cognitiva. A mensuração é realizada por meio da somatória dos domínios e a pontuação total } \\
\text { varia de zero (pior) até } 23 \text { (melhor). }\end{array}$ & $\begin{array}{l}E 15, E 16, E 17, \\
E 18, E 19, E 21 \\
E 23, E 26, E 27 \\
\text { E32 }\end{array}$ \\
\hline $\begin{array}{l}\text { Nociception Coma } \\
\text { Scale-Revised (NCS-R) }\end{array}$ & $\begin{array}{l}\text { A NCS-R é avaliada pelas respostas de expressão motora, verbal e facial. Cada subescore varia } \\
\text { de zero a três pontos, e a pontuação total varia de zero a } 9 \text { pontos A mensuração é realizada } \\
\text { por meio da somatória dos domínios. }\end{array}$ & $\begin{array}{l}\text { E17, E19, E22, } \\
\text { E33 }\end{array}$ \\
\hline $\begin{array}{l}\text { Glasgow Coma Scale } \\
\text { (GCS) }\end{array}$ & $\begin{array}{l}\text { A GCS é avaliada pelos seguintes domínios: abertura ocular }-1 \text { a } 4 \text { pontos; resposta verbal - } \\
1 \text { a } 5 \text { pontos; e a resposta motora }-1 \text { a } 6 \text { pontos. A mensuração é realizada por meio da } \\
\text { somatória dos domínios sendo classificada como leve }>13 \text { pontos, moderado } 9 \text { a } 12 \text { pontos e } \\
\text { grave ou severa < 8pontos. }\end{array}$ & $\begin{array}{l}\text { E15, E22, E24, } \\
\text { E20, E29, E28, } \\
\text { E31 }\end{array}$ \\
\hline $\begin{array}{l}\text { Full Outline of } \\
\text { Unresponsiveness } \\
\text { (FOUR) }\end{array}$ & $\begin{array}{l}\text { A FOUR é avaliada pelos domínios: abertura ocular, resposta motora, reflexos tronco } \\
\text { encefálicos e padrão respiratório, ambos pontuados de } 0 \text { a } 4 \text { pontos, sendo } 0 \text { a pior pontuação } \\
\text { e } 4 \text { a melhor. A pontuação total varia de } 0 \text { a } 16 \text { pontos e a mensuração é realizada por meio } \\
\text { da somatória dos domínios. }\end{array}$ & $\begin{array}{l}E 15, \quad E 24, \quad E 20 \\
E 29, E 28, E 31, E 2\end{array}$ \\
\hline $\begin{array}{l}\text { Brief Post-Coma Scale } \\
\text { (BPCS) }\end{array}$ & 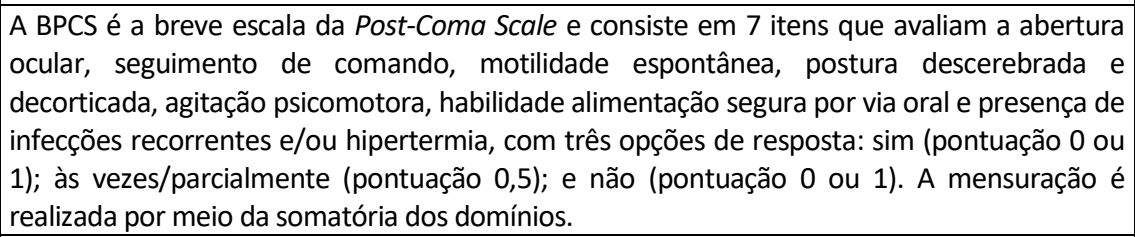 & E18 \\
\hline $\begin{array}{l}\text { Disability Rating Scale } \\
\text { (DRS) }\end{array}$ & $\begin{array}{l}\text { A DRS avalia o prejuízo do nível de consciência, incapacidade da capacidade cognitiva de } \\
\text { autocuidado e deficiência psicossocial e é avaliada como nenhum - } 0 \text { pontos; suave }-1 \text { ponto, } \\
\text { parcial - 2-3 pontos; moderado }-4-6 \text { pontos; moderadamente grave }-7-11 \text { pontos; grave - } \\
\text { 12-16 pontos; extremamente grave }-17-21 \text { pontos; estado vegetativo }-22-24 \text { pontos e; } \\
\text { estado vegetativo extremo - } 25-29 \text { pontos e a mensuração é realizada por meio da somatória } \\
\text { dos domínios. }\end{array}$ & E18 \\
\hline $\begin{array}{l}\text { Level of Cognitive } \\
\text { Functioning (LCF) }\end{array}$ & $\begin{array}{l}\text { A LCF avalia o nível de consciência e gera uma classificação do paciente em um dos oito níveis: } \\
1 \text { - sem resposta; } 2 \text { - generalizada; } 3 \text { - localizado; } 4 \text { - confuso-agitado; } 5 \text { - confuso, } \\
\text { inapropriado, não agitado; } 6 \text { - apropriado confuso; } 7 \text { - apropriado automaticamente; } 8 \text { - } \\
\text { finalidade apropriada. }\end{array}$ & E18 \\
\hline $\begin{array}{l}\text { Glasgow Outcome } \\
\text { Scale (GOS) }\end{array}$ & $\begin{array}{l}\text { A GOS é utilizada em pacientes com lesões cerebrais e caracteriza-o em } 5 \text { níveis, de acordo } \\
\text { com as sequelas sendo eles: falecido }-1 \text { ponto; estado vegetativo }-2 \text { pontos; incapacidade } \\
\text { severa - } 3 \text { pontos; incapacidade moderada - } 4 \text { pontos; e boa recuperação }-5 \text { pontos. }\end{array}$ & E18 \\
\hline
\end{tabular}

FIGURA 3: Métodos de avaliação do nível de consciência apresentados pelas escalas identificadas na amostra. Uberaba, MG, Brasil, 2020.

\section{DISCUSSÃO}

A totalidade de manuscritos que compuseram a amostra da presente pesquisa configura-se como estudos na língua inglesa, a maioria publicada no ano de 2012 e em 16 periódicos internacionais diferentes. Não foram identificados estudos nacionais que atendessem aos critérios de inclusão, o que demonstra a importância de aprofundamento científico neste âmbito, no Brasil ${ }^{34}$.

O tipo de estudo mais preponderante na amostra selecionada foi o metodológico, com destaque para a elaboração e validação de instrumentos para identificar e avaliar o nível de consciência de pacientes adultos e idosos. Estudos metodológicos possibilitam compreender o procedimento de validação de conteúdo e são essenciais para a utilização na construção de medidas e instrumentos confiáveis na área de Enfermagem, os quais subsidiam o conhecimento para uma prática mais segura ${ }^{35}$.

A maioria dos estudos apresentou baixo nível de evidência -6 , o que deve servir de incentivo para o desenvolvimento de novos estudos com delineamentos mais robustos que permitam produzir melhores níveis de evidência ${ }^{36}$. 
Estudos experimentais são indispensáveis para a prática clínica, pois, permitem a redução de riscos, prevenção, tratamento, resolução ou gerenciamento de um problema de saúde, atingindo o propósito desejado e podendo incluir ausência, resolução, gerenciamento bem-sucedido do problema ou não desenvolvimento de complicações ${ }^{36}$.

Cabe ressaltar que este estudo confere ineditismo à ciência em saúde e em enfermagem por sintetizar as evidências científicas existentes sobre as escalas que avaliam o nível de consciência, seus domínios e os mecanismos de avaliação, contribuindo para a estruturação de um arcabouço de conhecimento que direciona para as melhores práticas e decisões clínicas neste âmbito.

Oito escalas foram identificadas nesta revisão de literatura. Dentre estas, destacou-se a Coma Recovery ScaleRevised, abordada por oito dos estudos. Pesquisas sobre a Coma Recovery Scale-Revised apontaram sua confiabilidade e precisão em cenários diversificados para avaliar a consciência, considerando-a uma ferramenta de medição psicometricamente sólida e robusta ${ }^{15,16,22,23,25-27,32}$.

Corrobora com esta afirmação estudo de revisão integrativa da literatura que objetivou identificar artigos científicos que abordavam escalas para a avaliação do nível de consciência especificamente em pacientes com trauma cranioencefálico, evidenciando que a Coma Recovery Scale-Revised é confiável e assertiva para este fim, pela sua potencialidade em reconhecer o estado vegetativo e especificidade em avaliar a resposta neurocomportamental do paciente, entretanto, possui fragilidades por não avaliar o padrão respiratório, tornando-a inaplicável em pacientes em uso de $\mathrm{VMI}^{6}$.

Mesmo que a Coma Recovery Scale-Revised tenha sido largamente apontada pelos estudos que compuseram a amostra da presente revisão, observou-se que, nacionalmente, a adoção da Glasgow Coma Scale é comum e culturalmente bastante utilizada na prática clínica por demostrar-se potencialmente capaz de identificar disfunções neurológicas e acompanhar a evolução do nível de consciência, predizer prognóstico e padronizar a linguagem entre os profissionais de saúde ${ }^{37}$.

No que se refere às especificidades, a Glasgow Coma Scale possui uma avaliação ampla da resposta verbal, o que difere de outras escalas, no entanto, é importante enfatizar que essa escala possui fragilidades por não ser confiável na avaliação do nível de consciência de pacientes, sedados, afásicos, com uso de cânula endotraqueal e VMI ${ }^{6}$.

Diante do exposto, a adoção da escala denominada Full Outline of Unresponsiveness foi a mais indicada pelos estudos desta revisão por garantir um alto nível de precisão na mensuração do nível de consciência de pacientes graves em $\mathrm{VMI}^{20,24,28-31}$.

Estudo de validação da escala Full Outline of Unresponsiveness para a versão chinesa indicou este instrumento para a avaliação do nível de consciência e predição da mortalidade de pacientes em grave estado, devido à sua confiabilidade e por ser considerado de fácil aplicação ${ }^{33}$.

Revisão sistemática realizada em setembro de 2017 enfatiza a confiabilidade e potencialidade da escala Full Outline of Unresponsiveness em predizer a mortalidade de pacientes críticos, além de avaliar pacientes em uso VMI ${ }^{38}$. Ressalta-se que a referida escala tem como especificidade a capacidade de avaliar o padrão respiratório e os reflexos do tronco encefálico e com mais acurácia do que outros instrumentos, na avaliação da consciência de pacientes sedados, mesmo que não obtenha $100 \%$ de confiabilidade nesta condição, o que pode representar uma fragilidade ${ }^{6}$.

A literatura aponta que este mecanismo de identificação e avaliação por meio da somatória de domínios como a abertura ocular, resposta motora, resposta verbal, padrão respiratório e reflexos tronco encefálicos de pacientes adultos e idosos e adotados pelas escalas é efetivo e considerado confiável para predizer a gravidade indicada pelo nível de consciência desta população 20,24,28-31.

Em suma, observou-se que a descrição de uma escala ideal para a avaliação do nível de consciência é àquela capaz de identificar com precisão este parâmetro, que seja de fácil manuseio, tenha um alto nível de concordância interobservador, reprodutibilidade e valor preditivo e preveja rapidamente a deterioração do paciente, a morbidade e a mortalidade, além de ser aplicável ao maior número de pacientes ${ }^{6,39}$.

\section{Limitações do estudo}

A principal limitação identificada neste estudo foi o baixo nível de evidência dos achados e a falta de clareza na descrição dos mecanismos para mensurar o nível de consciência e no percurso metodológico dos estudos selecionados, o que dificultou a compreensão e identificação da maneira com que os estudos foram conduzidos e a forma de avaliação das escalas apresentadas. 


\section{CONCLUSÃO}

Os instrumentos elencados para avaliar o nível de consciência de adultos e idosos foram: Coma Recovery ScaleRevised, Nociception Coma Scale-Revised, Glasgow Coma Scale, Full Outline of Unresponsiveness, Brief Post-Coma Scale, Disability Rating Scale, Level of Cognitive Functioning, Glasgow Outcome Scale.

A Coma Recovery Scale-Revised foi a mais abordada pelos estudos, porém, a escala Full Outline of Unresponsiveness foi considerada a mais confiável, fidedigna, rápida e prática, para mensurar e avaliar o nível de consciência de pacientes adultos e idosos, principalmente por abordar pacientes em estado crítico e em ventilação mecânica invasiva. As escalas, em geral, são semelhantes em seus mecanismos de avaliação do nível de consciência e abordam principalmente, a somatória de critérios como a abertura ocular, resposta motora, resposta verbal, padrão respiratório e reflexos tronco encefálicos, indicando o pior estado do paciente para uma menor pontuação final e, para maior pontuação, o melhor estado.

A originalidade que acompanhou todas as etapas do presente estudo, desde a sua concepção conferem ineditismo ao mesmo. Destaca-se que este contribui para o ensino, pesquisa e assistência em saúde, a priori por compilar evidências cientificas sobre as principais escalas para identificar o nível de consciência de adultos e idosos e apresentar os seus mecanismos de avaliação, o que pode sustentar a melhor tomada de decisão para a prática clínica, para implantações, utilização e treinamentos e desenvolvimento de novas pesquisas cientificas, metodologicamente bemelaboradas, que se proponham a compará-las, validá-las e comparar a sua efetividade.

\section{REFERÊNCIAS}

1. Patel N, Nandakumar N, Heaton PA, Le Huray V, Paul SP. Decreased level of consciousness in a child: recognition and management. Br. J. Community Nurs. [Internet], 2020 [cited 2020 Oct 22]; 29(4):204-10. DOI: http://dx.doi.org/10.12968/bjon.2020.29.4.204.

2. Damiani D. Disorders of Consciousness: Practical Management in an Emergency Room. Arq. Bras. Neurocir. [Internet], 2019 [cited 2020 Oct 20]; 38(04):263-71. DOI: http://dx.doi.org/10.1055/s-0036-1594251.

3. Barros WCTS, Sasso GTMS, Alvarez AG, Ramos SF, Martins SR. Aplicativo para avaliação do nível de consciência em adultos: produção tecnológica em enfermagem. Cogitare enferm. [Internet], 2019 [cited 2020 Oct 22]; 24:e60338. DOI: http://dx.doi.org/10.5380/ce.v24i0.60338.

4. Oliveira DMP, Pereira CU, Freitas ZMP. Neurological evaluation about nursing knowledge of the patient with traumatic brain injury. Rev. Enferm. UFPE On Line [Internet], 2016 [cited 2020 Oct 22]; 10(sup.5):4249-54. Available from: https://periodicos.ufpe.br/revistas/revistaenfermagem/article/viewFile/11170/12702.

5. Madeira JC, Rodrigues IR, Pinheiro GFA, Alves AR, Sousa AAS. Elaboration of an Instrument to Systematize Nursing Care in a Vascular Cerebral Accident Unit. Res, Soc. Dev. [Internet], 2020 [cited 2020 Oct 06]; 9(10):e2889108532. DOI: https://doi.org/10.33448/rsd-v9i10.8532.

6. Oliveira DMP, Pereira CU, Freitas ZMP. Scales for evaluating the level of consciousness in trauma brain injury and their relevance to nursing practices. Arq. Bras. Neurocir. [Internet], 2014 [cited 2020 Oct 06]; 33(1):22-32. Available from: http://files.bvs.br/upload/S/0103-5355/2014/v33n1/a4284.pdf.

7. Gardona RGB, Barbosa DA. The importance of clinical practice supported by health assessment tools. Rev. Bras. Enferm. [Internet], 2018 [cited 2020 Oct 06]; 71(4):1815-6. DOI: http://dx.doi.org/10.1590/0034-7167-2018710401.

8. Souza AC, Alexandre NMC, Guirardello EB. Propriedades psicométricas na avaliação de instrumentos: avaliação da confiabilidade e da validade. Epidemiol Serv. Saúde [Internet], 2017 [cited 2020 sept 08]; 26(3):649-59. DOI: http://dx.doi.org/10.5123/S1679-49742017000300022.

9. Mendes KDS, Silveira RCCP, Galvão CM. Revisão integrativa: método de pesquisa para a incorporação de evidências na saúde e na enfermagem. Texto Contexto Enferm. [Internet], 2008 [cited 2020 Apr 10]; 17(4):758-64. DOI: https://doi.org/10.1590/S0104-07072008000400018.

10. Sousa LMM, Marques JM, Firmino CF, Frade F, Valentim OS, Antunes AV. Modelos de formulação da questão de investigação na prática baseada na evidência. Rev. Invest. Enferm. [Internet], 2018 [cited 2020 Oct 06]; 31-9. Available from: https://repositorio-cientifico.essatla.pt/bitstream/20.500.12253/1287/1/artigo31-39.pdf.

11. Ouzzani M, Hammady H, Fedorowicz Z, Elmagarmid A. Rayyan - a web and mobile app for systematic reviews. Syst Rev [Internet], 2016 [cited 2020 Apr 10]; 5(1):210. DOI: https://doi.org/10.1186/s13643-016-0384-4.

12. Ursi ES, Galvão CM. Perioperative prevention of skin injury: an integrative literature review. Rev. Latino-Am. Enfermagem [Internet], 2006 [cited 2020 Nov 4]; 14(1):124-31. DOI: http://dx.doi.org/10.1590/S0104-11692006000100017.

13. Bindman $A B$. The agency for healthcare research and quality and the development of a learning health care system. JAMA Intern. Med. [Internet], 2017 [cited 2020 Nov 4]; 177(7):909-10. DOI: http://dx.doi.org/10.1001/jamainternmed.2017.2589.

14. Page MJ, McKenzie JE, Bossuyt PM, Boutron I, Hoffmann TC, Mulrow CD, et al. The PRISMA 2020 statement: an updated guideline for reporting systematic reviews. BMJ [Internet]. 2020 [cited 2020 Nov 4]; 372:n71. DOI: https://doi.org/10.1136/bmj.n71.

15. Wang F, He Y, Qu J, Cao Y, Liu Y, Li F, et al. A brain-computer interface based on three-dimensional stereo stimuli for assisting clinical object recognition assessment in patients with disorders of consciousness. IEEE Trans. Neural Syst. Rehabilitation Eng. [Internet], 2019 [cited 2020 Nov 6]; 27(3):507-13. DOI: http://dx.doi.org/10.1109/TNSRE.2019.2896092. 
16. Riganello F, Chatelle C, Schnakers C, Laureys S. Heart Rate Variability as an indicator of nociceptive pain in disorders of consciousness? J. Pain Symptom. Manag. [Internet], 2019 [cited 2020 Nov 20]; 57(1):47-56. DOI: http://dx.doi.org/10.1016/j.jpainsymman.2018.09.016.

17. Bagnato S, Boccagni C, Sant'Angelo A, Alito A, Galardi G. Pain assessment with the revised nociception coma scale and outcomes of patients with unresponsive wakefulness syndrome: results from a pilot study. Neurol. Sci. [Internet], 2018 [cited 2020 Nov 3]; 39(6):1073-77. DOI: http://dx.doi.org/10.1007/s10072-018-3330-5.

18. Formisano R, Aloisi M, losa $M$, Contrada $M$, Rizza $F$, et al. A new tool to assess responsiveness in disorders of consciousness (DoC): a preliminary study on the Brief Post-Coma Scale (BPCS). Neurol. Sci. [Internet], 2018 [cited 2020 Nov 30]; 39(9):1651-56. DOI: http://dx.doi.org/10.1007/s10072-018-3466-3.

19. Sattin D, Schnakers C, Pagani M, Arenare F, Devalle G, Giunco F, et al. Evidence of altered pressure pain thresholds in persons with disorders of consciousness as measured by the Nociception Coma Scale-Italian version. Neuropsychol. Rehabil. [Internet], 2018 [cited 2020 Nov 15]; 28(8):1295-310. DOI: http://dx.doi.org/10.1080/09602011.2017.1290532.

20. Momenyan S, Mousavi SM, Dadkhahtehrani T, Sarvi F, Heidarifar R, Kabiri F, et al. Predictive validity, and inter-rater reliability of the Persian version of full outline of unresponsiveness among unconscious patients with traumatic brain injury in an intensive care unit. J. Neurocrit. Care [Internet], 2017[cited 2020 Nov 20]; 27(2):229-36. DOI: http://dx.doi.org/10.1007/s12028-0160324-0.

21. Pignat JM, Mauron E, Jöhr J, Gilart de Keranflec'h C, Van De Ville D, Preti MG, et al. Outcome prediction of consciousness disorders in the acute stage based on a complementary motor behavioural tool. PLOS ONE [Internet], 2016 [cited 2020 Nov 20]; 11(6):e0156882. DOI: http://dx.doi.org/10.1371/journal.pone.0156882.

22. Chatelle C, De Val MD, Catano A, Chaskis C, Seeldrayers P, Laureys S, et al. Is the nociception coma scale-revised a useful clinical tool for managing pain in patients with disorders of consciousness? Clin. J. Pain [Internet], 2016 [cited 2020 nov 30]; 32(4):32126. DOI: http://dx.doi.org/10.1097/ajp.0000000000000259.

23. Cortese M, Riganello F, Arcuri F, Pugliese M, Lucca L, Dolce G, Sannita W. Coma recovery scale-r: variability in the disorder of consciousness. BMC Neurol. [Internet], 2015 [cited 2020 Nov 30]; 15(1):186. DOI: http://dx.doi.org/10.1186/s12883-015-0455-5.

24. Peng J, Deng Y, Chen F, Zhang X, Wang X, Zhou Y, et al. Validation of the Chinese version of the FOUR score in the assessment of neurosurgical patients with different level of consciousness. BMC Neurol. [Internet], 2015 [cited 2020 Nov 10]; 15(1):254. DOI: http://dx.doi.org/10.1186/s12883-015-0508-9.

25. La Porta F, Caselli S, lanes AB, Cameli O, Lino M, Piperno R, et al. Can we scientifically and reliably measure the level of consciousness in vegetative and minimally conscious states? Rasch analysis of the coma recovery scale revised. Arch. Phys. Med. Rehabil. [Internet], 2013 [cited 2020 Dec 07]; 94(3):527-35. DOI: http://dx.doi.org/10.1016/j.apmr.2012.09.035.

26. Lechinger J, Bothe K, Pichler G, Michitsch G, Donis J, Klimesch W, et al. CRS-R score in disorders of consciousness is strongly related to spectral EEG at rest. J. Neurol. [Internet], 2013 [cited 2020 Dec 07]; 260(9):2348-56. DOI: http://dx.doi.org/10.1007/s00415-013-6982-3.

27. Noé E, Olaya J, Navarro MD, Noguera P, Colomer C, García-Panach J, et al. Behavioral recovery in disorders of consciousness: a prospective study with the Spanish version of the coma recovery scale-revised. Arch. Phys. Med. Rehabil. [Internet], 2012 [cited 2020 Nov 6]; 93(3):428-33. DOI: http://dx.doi.org/10.1016/j.apmr.2011.08.048.

28. Kocak Y, Ozturk S, Ege F, Ekmekci AH. A useful new coma scale in acute stroke patients: four score. Anaesth. Inten. Care [Internet], 2012 [cited 2020 Nov 20]; 40(1):131-36. DOI: http://dx.doi.org/10.1177/0310057x1204000115.

29. Kramer AA, Wijdicks EFM, Snavely VL, Dunivan JR, Naranjo LLS, Bible S, et al. A multicenter prospective study of interobserver agreement using the Full Outline of Unresponsiveness score coma scale in the intensive care unit. Crit. Care Med. [Internet], 2012 [cited 2020 nov 20]; 40(9):2671-76. DOI: http://dx.doi.org/10.1097/ccm.0b013e318258fd88.

30. Marcati E, Ricci S, Casalena A, Toni D, Carolei A, Sacco S. Validation of the Italian version of a new coma scale: the four score. Intern. Emerg. Med. [Internet], 2011 [cited 2020 dez 07]; 7(2):145-52. DOI: http://dx.doi.org/10.1007/s11739-011-0583-x.

31. Bruno MA, Ledoux D, Lambermont B, Damas F, Schnakers C, Vanhaudenhuyse A, et al. Comparison of the full outline of unresponsiveness and Glasgow liege scale/Glasgow coma scale in an intensive care unit population. J. Neurocrit. Care [Internet], 2011 [cited 2020 nov 3]; 15(3):447-53. DOI: http://dx.doi.org/10.1007/s12028-011-9547-2.

32. Sacco S, Altobelli E, Pistarini C, Cerone D, Cazzulani B, Carolei A. Validation of the Italian version of the Coma Recovery ScaleRevised (CRS-R). Brain Inj. [Internet], 2011 [cited 2020 nov 15]; 25(5):488-95. DOI: http://dx.doi.org/10.3109/02699052.2011.558043.

33. Schnakers C, Chatelle C, Vanhaudenhuyse A, Majerus S, Ledoux D, Boly M, Laureys S. The Nociception Coma Scale: A new tool to assess nociception in disorders of consciousness. Pain [Internet], 2010 [cited 2020 Nov 15]; 148(2):215-19. DOI: http://dx.doi.org/10.1016/j.pain.2009.09.028.

34. Dalla Nora CR, Zoboli E, Vieira MM. Validation by experts: importance in translation and adaptation of instruments. Rev. Gaúcha Enferm. [Internet], 2017 [cited 2020 Nov 10]; 38(3):e64851. DOI: http://dx.doi.org/10.1590/1983-1447.2017.03.64851.

35. Medeiros RKS, Ferreira JMA, Pinto DPSR, Vitor AF, Santos VEP, Barichello E. Pasquali's model of content validation in Nursing researches. Rev. Enf. Ref. [Internet], 2015 [cited 2020 Apr 07]; serIV (4):127-35. DOI: http://dx.doi.org/10.12707/RIV14009.

36. Dutra HS, Reis VN. Experimental and quasi-experimental study designs: definitions and challenges in nursing research. Rev. enferm. UFPE on line [Internet], 2016 [cited 2020 Apr 07]; 10(6):2230-41. DOI: https://periodicos.ufpe.br/revistas/revistaenfermagem/article/view/11238.

37. Santos WC, Vancini-Campanharo CR, Lopes MCBT, Okuno MFP, Batista REA. Assessment of nurse's knowledge about Glasgow coma scale at a university hospital. Einstein [Internet], 2016 [cited 2020 Apr 09]; 14(2):213-8. DOI: https://doi.org/10.1590/S1679-45082016AO3618. 
38. Almojuela A, Hasen M, Zeiler FA. The Full Outline of UnResponsiveness (FOUR) Score and its use in outcome prediction: a scoping systematic review of the adult literature. J. Neurocrit. Care [Internet], 2019 [cited 2020 Apr 09]; 31(1):162-75. DOI: https://doi.org/10.1007/s12028-018-0630-9.

39. Luna AA, Entringer AP, Silva RCL. Prevalence of under-diagnosis of delirium among patients in an intensive care unit. Rev. enferm. UERJ [Internet], 2016 [cited 2021 Jun 16]; 24(1):1-5. DOI: http://dx.doi.org/10.12957/reuerj.2016.6238. 